\title{
Definição da taxa de infiltração para dimensionamento de sistemas de irrigação por aspersão
}

\author{
Carlos B. M. Calheiros ${ }^{1}$, Filipe J. C. Tenório ${ }^{2}$, Jorge L. X. L. Cunha ${ }^{3}$, Edson T. da Silva ${ }^{3}$, Djair F. da Silva ${ }^{3}$ \& José A. C. da Silva ${ }^{3}$
}

\begin{abstract}
RESUMO
Objetivou-se, neste trabalho, determinar, dentre as técnicas nele utilizadas, a mais adequada para a definição da taxa de infiltração visando ao dimensionamento de sistemas de irrigação por aspersão. Obtiveram-se os dados de infiltração de água no solo segundo uma malha com espaçamentos variáveis entre pontos de ensaios de infiltração de 1,$5 ; 3 ; 6 ; 12$; 24; 48 e 96 m, totalizando 97 pontos de ensaio. As discussões relativas aos resultados obtidos pelas técnicas de definição - moda, média harmônica, mediana, média geométrica, média aritmética simples, média quadrática, média cúbica e média biquadrática, foram alicerçadas em um número maior de atributos, tais como: técnicas de análises estatísticas clássicas e geoestatísticas. O modelo de equação que descreve a taxa de infiltração utilizada foi a de Kostiakov. A média aritmética simples, consagrada como a técnica mais utilizada para definição da taxa de infiltração para dimensionamentos, não deve ser utilizada, sendo a mediana e a média geométricas mais recomendadas em cultivos com cana-de-açúcar; contudo, a mediana apresentou um número maior de atributos desejáveis. Na área estudada o valor da taxa de infiltração para dimensionamentos deve ser adotado entre 30 e $44 \mathrm{~mm} \mathrm{~h}^{-1}$, em se tratando da $\mathrm{Md} \pm \mathrm{s}^{2}(\hat{\mathrm{m}})$.
\end{abstract}

Palavras-chave: água no solo, cana-de-açúcar, disponibilidade hídrica

\section{Definition of the infiltration rate for the design of sprinkler irrigation system}

\begin{abstract}
The aim of this research was to determine which technique is the most appropriate for the infiltration rate definition for the design of sprinkler irrigation systems. The water infiltration data was obtained according to a net having variable spaces between infiltration test points of 1.5, 3, 6, 12, 24, 48 and $96 \mathrm{~m}$, summing 97 test points. The discussions of results obtained by means of definition techniques, i.e. mode, harmonic mean, median, geometric mean, and simple arithmetic mean, square mean, cubic mean and bisquare were based on a larger number of attributes, such as techniques for classical statistics and geostatistical analysis. The equation model for the infiltration rate used was the Kostiakov. The simple arithmetic mean, which is considered the most used technique for definition of infiltration rate for sprinkler irrigation project, should not be used. The median and the geometric mean are the most recommended in sugar cane cultivation, however the median presented a higher number of desirable attributes. In the studied area, the adopted value of the infiltration rate for design must be between 30 and $44 \mathrm{~mm} \mathrm{~h}^{-1}$, when considering $\mathrm{Md} \pm \mathrm{s}^{2}(\hat{\mathrm{m}})$.
\end{abstract}

Key words: soil water, sugarcane, water availability

1 Engenharia de Líquidos - CECA/UFAL. CEP 57000-000, Rio Largo, AL. Fone: (82) 9912-1183. E-mail: cbmcalheiros@yahoo.com.br

2 IBAMA-MA, Av. Jaime Távora, n² 25, CEP 65054-770, São Luis, MA. Fone: (82) 9972-2906. E-mail: filipe-carten@ig.com.br

${ }_{3}^{3}$ CECA/UFAL, CEP 57000-000, Rio Largo, AL. Fone: (82) 3261-1776. E-mails: jlxlcunha@yahoo.com.br; edsonnets@gmail.com; djair_felix@yahoo.com.br; jandrec@yahoo.com.br 


\section{INTRODUÇÃO}

A dinâmica da água no solo está diretamente relacionada à produção vegetal e seu conhecimento é de interesse fundamental para qualquer tomada de decisão sobre a exploração agrícola dos solos. Portanto, a melhor caracterização dos fatores que interferem nesta dinâmica se torna imprescindível (Lewis \& Powers, 1938). Segundo Brito (1994), no dimensionamento de um projeto de irrigação é indispensável dispor de informações relacionadas às variáveis climáticas, ao solo e à cultura. Relacionada com o solo, a principal característica é a velocidade básica de infiltração (Lima \& Silans, 1999), pois reflete a capacidade do solo em conduzir a água; no caso da irrigação por aspersão, a velocidade básica de infiltração determina a intensidade de precipitação máxima que poderá ser aplicada ao solo, evitando o escoamento superficial (Coelho et al., 2000b). Para Cichota et al. (2003) ela tem importância agronômica pelo seu papel na formação de enxurrada, agente erosivo, e na determinação de taxas viáveis de irrigação.

Tão importante como a determinação da velocidade básica de infiltração do solo, é a definição da velocidade de infiltração para projetos (VIP - valor representativo da velocidade básica de infiltração da água no solo de uma área onde será implantado e manejado um projeto de irrigação) que indica o objetivo e o interesse de cada situação de irrigação, principalmente no cultivo de cana-de-açúcar em solos de Tabuleiros Costeiros (Calheiros et al., 2003).

No Brasil, esta situação é observada divido à sua extensão territorial e à diversidade climática, onde se encontram as mais variáveis possíveis. Em regiões áridas e semi-áridas no Nordeste brasileiro, por exemplo, o manejo correto implica em práticas de economia de água e cuidados com problemas de salinidade. Tenório et al. (2008), verificaram que a economia no uso da água em projetos de irrigação por no uso da água em projetos de irrigação por ordem das técnicas utilizadas na de definição da VIP: MB, MC, MQ, MAS, MG, Md, MH e Mo. Já em regiões com excesso de umidade no solo, Norte brasileiro, o problema fundamental é a lixiviação de materiais no solo e em regiões em que a chuva é suficiente, Tabuleiros Costeiros, geralmente há problemas de distribuição que acarretam a existência de períodos de falta de água; nessas áreas é de suma importância obter a maior eficiência possível no uso da água pelas culturas (Parr \& Bertrand, 1960).

A obtenção da VIP representa a variabilidade do solo a ser irrigado, haja visto que a situação e a finalidade do projeto são etapas no contexto da agricultura irrigada que, na maioria das vezes, não tem a atenção necessária, tamanha a sua importância. Uma má definição dessa taxa de infiltração poderá: ocasionar falhas no dimensionamento de estruturas inerentes à irrigação por aspersão (Coelho et al., 2000a); diminuir a eficiência do sistema; aumentar os custos de operação, gastos de energia e consumo de água (líquido) e maximizar impactos ambientais, como erosão do solo, lixiviação de nutrientes e salinização, dentre outros.

Devido à deficiência de estudos consistentes no Brasil referentes às técnicas de definição da VIP ( Tenório \& Calheiros, 2004), este trabalho tem como objetivo determinar, dentre essas técnicas, a velocidade de infiltração para projetos de irrigação por aspersão, que se apresenta mais eficiente levando-se em consideração a grande variabilidade espacial existente nos solos estudados, e a situação de irrigação em Tabuleiros Costeiros cultivados com cana-deaçúcar, visando que este sirva de base para futuros estudos regionalizados e venha a atender aos objetivos diversos e específicos de cada situação de irrigação.

\section{MATERIAL E MÉTODOS}

\section{Descrição geral da área experimental}

O experimento foi realizado em área de Tabuleiros Costeiros com altitude média de 103 m e coordenadas $9^{\circ} 44^{\prime} 48^{\prime \prime} \mathrm{S}$ e $35^{\circ} 58^{\prime} 04^{\prime}$ 'W, localizado na Fazenda Charles I, pertencente à Companhia Açucareira Central Sumaúma, localizada no município de Marechal Deodoro, Estado de Alagoas. O relevo da região é caracterizada por grandes áreas planas ligadas à presença de depressões fechadas; sua vegetação primária se caracteriza por florestas tropicais subperenifólia (Mata Atlântica), formação densa e alta (20-30 m), muito rica em espécies; no entanto, esta vegetação só é encontrada, atualmente, em áreas de relevo mais acidentado já que as áreas planas estão sendo exploradas com a cultura da cana-de-açúcar (Santos, 2004). O clima da região é tropical quente e úmido com estação seca de primavera-verão e chuvosa de outono-inverno. Os solos da área, conforme um pré-levantamento realizado pelo grupo de pedologia e classificação da UFAL e baseado no Sistema Brasileiro de Classificação de Solos (EMBRAPA, 1999), apresenta grande heterogeneidade entre as classes de Argissolos e Espodossolos, com predominância da primeira.

\section{Plano amostral}

Após o corte, a colheita e todos os tratos culturais realizados, demarcou-se a área com auxílio de miras e trena métrica, constituindo uma malha quadriculada (grid) com espaçamentos variáveis entre pontos de ensaios, de 1,5; 3,0; 12,$0 ; 6,0 ; 24,0 ; 48,0$ e $96,0 \mathrm{~m}$, totalizando 97 pontos de ensaio (Figura 1).

\section{Caracterização da curva de velocidade de infiltração e da velocidade de infiltração para projetos}

A técnica utilizada para leitura da infiltração da água no solo foi a do infiltrômetro de anéis concêntricos (IANC), (Bower, 1986; Coelho et al., 1992) o modelo adotado para caracterização da curva de infiltração foi o de Kostiakov , (Kostiakov, 1932; Leme, 1987).

Utilizaram-se oito técnicas para obtenção da velocidade de infiltração para projetos, com base nos valores das velocidades básicas de infiltração correspondente ao tempo de seis horas e trinta minutos de cada curva de infiltração. Obteve-se a média aritmética simples (MAS) através das equações: 


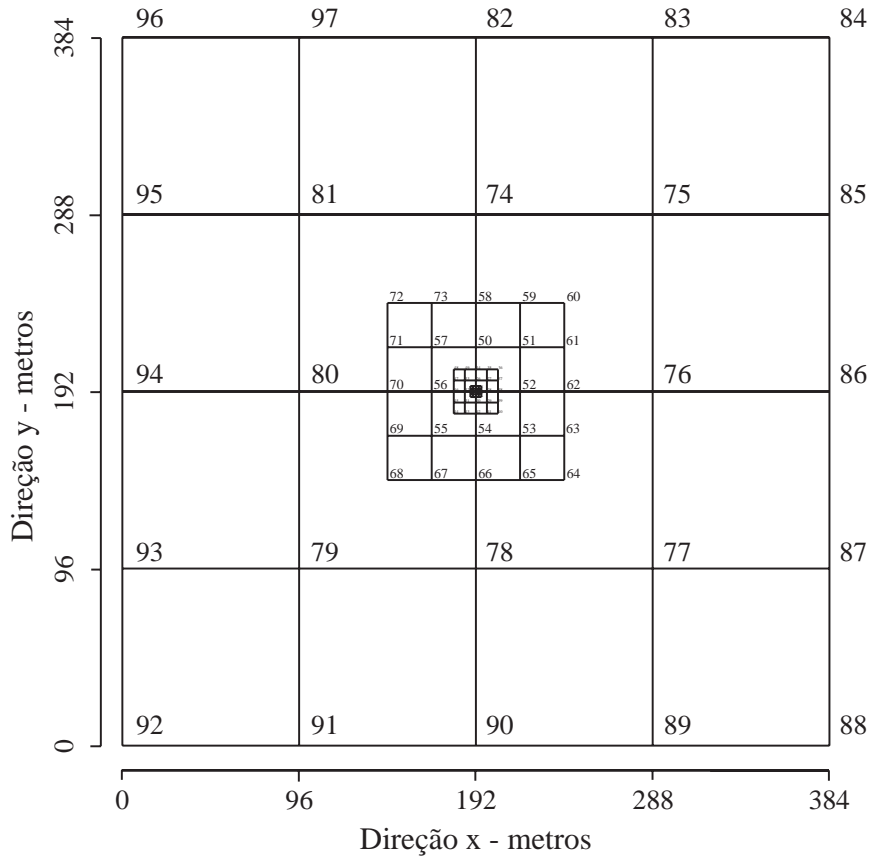

Figura 1. Plano amostral com a numeração dos ensaios, localizado na Fazenda Charles I, Usina Sumaúma, Marechal Deodoro, AL

$$
\begin{gathered}
\bar{X}=\frac{\sum_{i=1}^{n} X_{i}}{n} \\
\bar{X}=\frac{\sum_{i=1}^{n} \mathrm{Pm}_{i} f_{i}}{\sum_{i=1}^{n} f_{i}}
\end{gathered}
$$

Adotou-se a Eq. 2, uma vez que a VBI se trata de uma variável contínua e seus dados foram agrupados em uma distribuição de classes, sendo $\mathrm{Pm}_{\mathrm{i}}$ o ponto médio de cada classe e a frequência de cada classe.

A média geométrica (MG) foi utilizada no trabalho de Coelho et al. (1992) como técnica para obtenção da VIP, através da aplicação da Eq. 3, mas, neste trabalho, optou-se pela Eq. 4, já que a VBI é definida como variável contínua.

$$
\begin{gathered}
M G=\sqrt[n]{\prod_{i=1}^{n} x_{i}} \\
M G=\operatorname{antilog}\left[\frac{\sum_{i=1}^{n} \log P m_{i} \cdot f_{i}}{\sum_{i=1}^{n} f_{i}}\right]
\end{gathered}
$$

A Média harmônica (MH), é uma técnica pouco utilizada na obtenção da VIP, porém, mediante a propriedade de ter seu valor geralmente abaixo dos obtidos pela MG e MAS, Médici (1967); Conforme Pereira \& Tanaka (1984); Toledo \& Ovalle (1985), a MH foi utilizada pela aplicação da Eq. 5, de vez que é indicada para dados agrupados.

$$
\mathrm{MH}=\frac{\sum_{\mathrm{i}=1}^{\mathrm{n}} \mathrm{f}_{\mathrm{i}}}{\sum_{\mathrm{i}=1}^{\mathrm{n}} \frac{\mathrm{f}_{\mathrm{i}}}{\mathrm{Pm}_{\mathrm{i}}}}
$$

A Média quadrática (MQ) é uma técnica recente na obtenção da VIP, largamente utilizada na Estatística clássica para calcular a média dos desvios (desvio-padrão), ao invés da média dos valores originais (T oledo \& Ovalle, 1985). Na definição da VIP da área estudada neste trabalho se aplicou a equação:

$$
M Q=\sqrt{\frac{\sum_{i=1}^{n} \operatorname{Pm}_{1}^{2} \cdot f_{i}}{\sum_{i=1}^{n} f_{i}}}
$$

A Média cúbica (Mc) e a Média biquadrática (MB), são novas técnicas de definição da VIP e foram aplicadas através das Eqs. 7 e 8, respectivamente.

$$
\begin{aligned}
& M c=\sqrt[3]{\frac{\sum_{i=1}^{n} \mathrm{Pm}_{i}^{3} \cdot \mathrm{f}_{\mathrm{i}}}{\sum_{\mathrm{i}=1}^{\mathrm{n}} \mathrm{f}_{\mathrm{i}}}} \\
& M b=\sqrt[4]{\frac{\sum_{\mathrm{i}=1}^{\mathrm{n}} \mathrm{Pm}_{\mathrm{i}}^{4} \cdot \mathrm{f}_{\mathrm{i}}}{\sum_{\mathrm{i}=1}^{n} \mathrm{f}_{\mathrm{i}}}}
\end{aligned}
$$

Obteve-se a mediana (Md) através da fórmula seguinte:

$$
\mathrm{Md}=\mathrm{L}_{\mathrm{i}}+\left(\frac{\frac{\mathrm{n}}{2}-\sum \mathrm{f}^{\prime}}{\mathrm{f}_{\mathrm{Md}}}\right) \cdot \mathrm{C}_{\mathrm{Md}}
$$

donde:

$\mathrm{L}_{\mathrm{i}}$ - é o limite inferior da classe mediana, n o total de frequência

$\Sigma \mathrm{f}^{\prime}$ - soma de todas as frequências das classes inferiores à mediana,

$\mathrm{f}_{\mathrm{Md}}$ - frequência da classe mediana

$\mathrm{C}_{\mathrm{Md} \text { - amplitude da classe mediana. }}$

A moda (Mo) foi empregada pelo processo de Czuber, para definição da VIP:

$$
M o=L_{i M o}+\left(\frac{f_{M o}+f_{a}}{2 f_{M o}-\left(f_{a}+f_{p}\right)}\right) \cdot C_{M o}
$$

em que:

$\mathrm{L}_{\mathrm{iMo}}$ - limite inferior da classe modal

$f_{a}$ e $f_{p}$ - são as diferenças entre a frequência da classe modal e a das classes anteriores e posteriores, respectivamente

$\mathrm{f}_{\mathrm{Mo}}$ - frequência da classe modal

$\mathrm{C}_{\mathrm{Mo}}$ - amplitude da classe modal

\section{Análises descritivas e exploratórias}

Os valores das velocidades de infiltração correspondentes aos 97 ensaios realizados e as VIP das 8 técnicas de definição, foram submetidos às medidas de variabilidade de dados da estatística clássica: amplitude total (At), desvio médio (Dm), desvio-padrão (s), variância $\left(\mathrm{s}^{2}\right)$, erro padrão da média $(\mathrm{s}(\hat{\mathrm{m}}))$, coeficiente de variação $(\mathrm{CV})$, intervalo de 
confiança (IC), assimetria e curtose; efetuou-se também, a análise da distribuição de frequência dos valores para verificar se as distribuições seriam do tipo normal ou log nor mal. Para testar a hipótese de normalidade ou de log nor malidade dos valores da VBI dos ensaios, fez-se uso do teste W, Shapiro \& Wilk (1965) a 5\% de probabilidade e, com vistas à determinação da existência ou não da dependência espacial, usou-se o sistema de semivariogramas no qual se considerou o comportamento do conjunto de valores da VBI. As análises deste sistema foram realizadas através do programa computacional VARIOWIN-2.2.

Conhecendo-se o semivariograma da VBI, utilizou-se da técnica de interpolação de Krigagem. A VBI foi dividida nos mapas de contorno e superfície, em classes idênticas às classes da distribuição de frequências e a cada um se atribuiu uma cor de tonalidade azul e, quanto mais intensa a coloração maior também a VBI da classe; por outro lado, elaboram-se mapas identificando as áreas sujeitas a alagamento e a escoamento, na ocasião da utilização das VIP obtidas por cada uma das técnicas de definições estudadas, em que as áreas susceptíveis a alagamentos e escoamentos foram identificadas pela cor vermelha enquanto as áreas "não problemáticas", em tonalidade azul.

\section{RESULTADOS E DISCUSSÃO}

A velocidade de infiltração para projetos VIP calculada pela Mo teve o valor definido em 24,32 $\mathrm{mm} \mathrm{h}^{-1}$ (Tabela 1). Observa-se, com base na distribuição de frequência com os valores da VBI agrupados em classes (T abela 2), que a VIP calculada pela Mo se localiza na classe 1, a qual apresenta uma frequência de 50 valores da VBI do total de 97, correspondendo a $51,546 \%$ dos valores obtidos. Com relação ao $\mathrm{Dm}$, apresentou valor de 42,49 $\mathrm{mm} \mathrm{h}^{-1}$, configurando-se o menor entre as oito VIP (Tabela 1); já o seu FR contém 60,3\% dos valores da VI (Tabela 3 ).

Conclui-se, com base no exposto acima e na avaliação visual da área com possibilidade de ocorrência de escoamento (Figura 2), que a utilização da VIP definida pela Mo é satisfatória, recomendação feita também por Coelho et. al. (1992), ao afirmarem que as modas dos valores da VBI obtidas pelo IANC se aproximam da velocidade real de infiltração do terreno porém, se analisar -se o valor da assimetria e a curva de normalidade (T abela 1), a VIP
Tabela 2. Distribuição de frequência dos valores de VBI agrupados em classes

\begin{tabular}{|c|c|c|c|c|c|c|c|}
\hline \multirow{2}{*}{ Classe } & \multicolumn{2}{|c|}{ Valor } & \multirow{2}{*}{$F$} & \multirow{2}{*}{ FA } & \multirow{2}{*}{$\begin{array}{l}\text { FR } \\
\text { (\%) }\end{array}$} & \multirow{2}{*}{$\begin{array}{l}\text { FRA } \\
\text { (\%) }\end{array}$} & \multirow{2}{*}{ Pm } \\
\hline & mínimo & máximo & & & & & \\
\hline 1 & 3,33 & 38,1663 & 50 & 50 & 51,546 & 51,546 & 20,7481 \\
\hline 2 & 38,1663 & 73,0025 & 17 & 67 & 17,526 & 69,072 & 55,5844 \\
\hline 3 & 73,0025 & 107,8388 & 13 & 80 & 13,402 & 82,474 & 90,4206 \\
\hline 4 & 107,8388 & 142,6750 & 5 & 85 & 5,155 & 87,629 & 125,2569 \\
\hline 5 & 142,6750 & 177,5113 & 5 & 90 & 5,155 & 92,784 & 160,0931 \\
\hline 6 & 177,5113 & 212,3475 & 4 & 94 & 4,124 & 96,907 & 194,9294 \\
\hline 7 & 212,3475 & 247,1837 & 1 & 95 & 1,031 & 97,938 & 229,7656 \\
\hline 8 & 247,1837 & 282,0200 & 2 & 97 & 2,062 & 100 & 264,6018 \\
\hline
\end{tabular}

F - frequência; FA - frequência acumulada; FR - frequência relativa; FRA - frequência relativa acumulada e Pm - ponto médio da classe

Tabela 3. Intervalos de confiança das VIP e frequências dos intervalos de confiança, baseados na distribuição de frequência e no ponto médio das classes

\begin{tabular}{crrrr}
\hline \multirow{2}{*}{ TDV } & VIP & \multicolumn{2}{c}{ IC } & FR \\
\cline { 3 - 4 } mm h $^{-1}$ & \multicolumn{2}{c}{$\mathbf{9 9 , 9 9 \%}$} & \% \\
Mo & 24,32 & 0,0 & 49,08 & 60,3 \\
MH & 32,74 & 9,44 & 56,04 & 69,1 \\
Md & 37,12 & 14,47 & 59,77 & 69,1 \\
MG & 43,38 & 21,52 & 65,25 & 43,3 \\
MAS & 63,13 & 42,36 & 83,89 & 24,2 \\
MQ & 86,85 & 64,51 & 109,20 & 24,7 \\
MC & 103,04 & 78,06 & 128,02 & 18,6 \\
MB & 125,32 & 95,32 & 155,31 & 14,4 \\
\hline
\end{tabular}

TDV - técnicas de definição da velocidade de infiltração para projetos; VIP - velocidade de infiltração para projetos de irrigação por aspersão; IC - intervalo de confiança a 99,99\%; FR - frequência relativa da VBI no IC das VIP

obtida por esta técnica não será recomendada pois, segundo Toledo \& Ovalle (1985) na distribuição assimétrica, à direita, há predominância de valores superiores aos Mo, da decisão endossada pelos valores de s, s ${ }^{2}, \mathrm{~s}(\hat{\mathrm{m}})$ e CV (Tabela 1).

Utilizando-se a Média harmônica, encontrou-se o valor de $32,74 \mathrm{~mm} \mathrm{~h}^{-1}$ referente à VIP (Tabela 1). Esta velocidade se mantém na classe 1 da distribuição de frequência (Tabela 2), com uma FR de 51,546\% localizada, portanto, na mesma classe da VIP definida pela Mo; outro atributo que não permite decisão alguma sobre qual dessas duas VIP adotar, é o Dm, já que a diferença entre os dois foi de apenas $0,26 \mathrm{~mm} \mathrm{~h}^{-1}$.

Tabela 1. VIP e Estatística descritiva para as técnicas de definição da velocidade de infiltração para projetos

\begin{tabular}{|c|c|c|c|c|c|c|c|c|}
\hline Técnicas & $\begin{array}{c}\text { VIP } \\
\mathrm{mm} \mathrm{h}^{-1}\end{array}$ & At & $\mathrm{Dm}$ & s & $s(\hat{m})$ & $\begin{array}{c}\mathrm{CV} \\
\%\end{array}$ & \multicolumn{2}{|c|}{$\begin{array}{c}\text { IC } \\
99,99 \%\end{array}$} \\
\hline Mo & 24,32 & 278,69 & 42,49 & 5117,81 & 7,26 & 294,21 & 0,0 & 49,08 \\
\hline MH & 32,74 & 278,69 & 42,75 & 4529,02 & 6,83 & 205,57 & 9,44 & 56,04 \\
\hline $\mathrm{Md}$ & 37,12 & 278,69 & 42,88 & 4279,17 & 6,64 & 176,22 & 14,47 & 59,77 \\
\hline$M G$ & 43,38 & 278,69 & 43,08 & 3989,66 & 6,41 & 145,59 & 21,52 & 65,25 \\
\hline MAS & 63,13 & 278,69 & 46,33 & 3595,86 & 6,09 & 94,99 & 42,36 & 83,89 \\
\hline$M Q$ & 86,85 & 278,69 & 55,38 & 4164,76 & 6,55 & 74,30 & 64,51 & 109,20 \\
\hline Mc & 103,04 & 278,69 & 64,94 & 5205,71 & 7,33 & 70,02 & 78,06 & 128,02 \\
\hline $\mathrm{Mb}$ & 125,32 & 278,69 & 79,41 & 7503,70 & 8,80 & 69,12 & 95,32 & 155,31 \\
\hline
\end{tabular}

At - amplitude total; Dm - desvio médio; $s$ - desvio-padrão; $\mathrm{s}^{2}$ - variância; $s(\hat{m})$ - erro padrão da média; $\mathrm{CV}$ - coeficiente de variação; IC - intervalo de confiança 


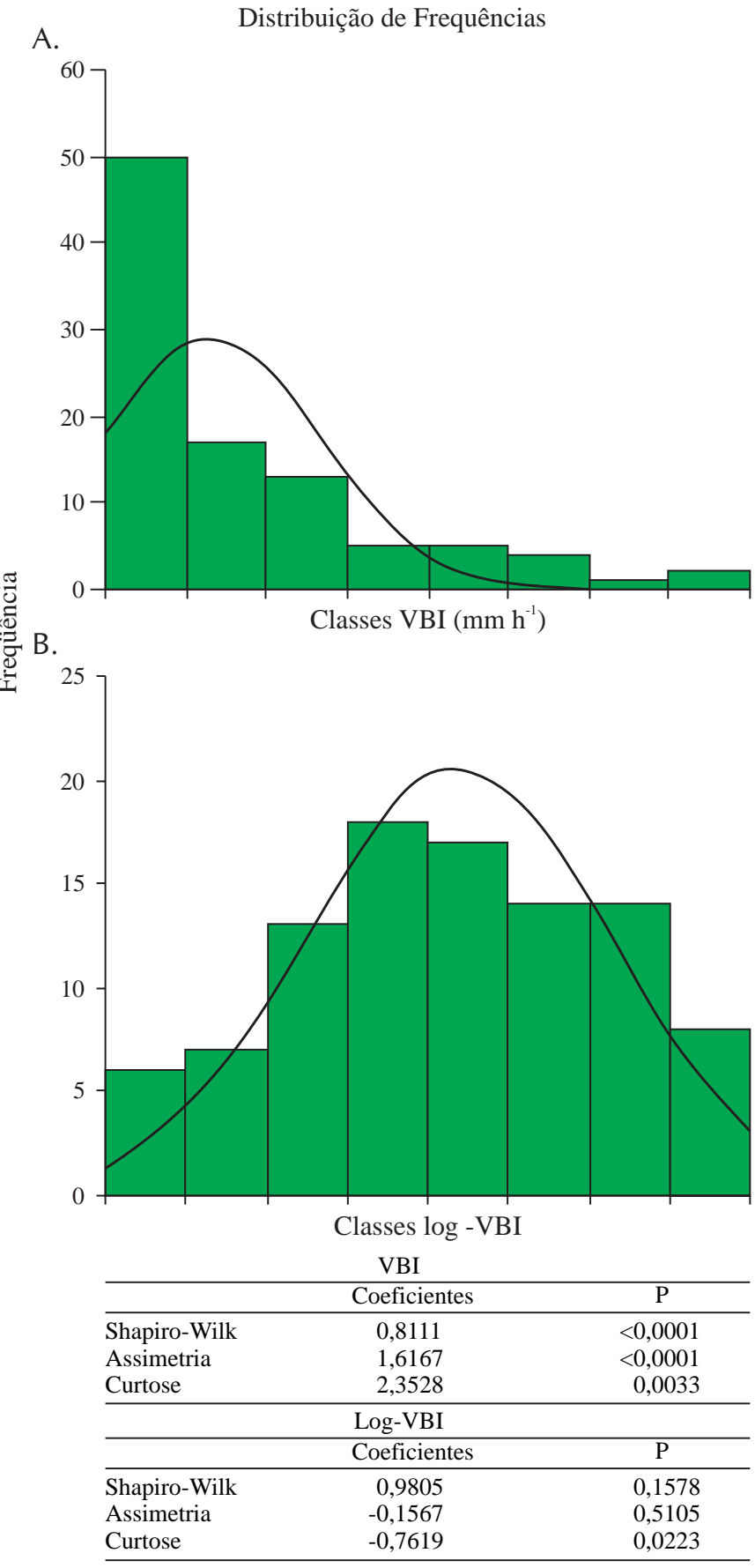

Figura 2. Distribuição de frequência com valores das classes de $\mathrm{VBI}\left(\mathrm{mm} \mathrm{h}^{-1}\right)$ (A) e de log-VBI, curva de normalidade e teste de Shapiro-Wilk a $p<0,05$ (B)

Embora a utilização da VIP encontrada pela MH apresente, quando comparada com a Mo, um pequeno acréscimo na área sujeita a escoamento, ela é mais precisa, haja vista que contém menor erro padrão da média $\mathrm{s}(\mathrm{m})$ ( $\mathrm{T}$ abela 1$)$; apresentou-se, também, mais precisa que as obtidas por Araújo Filho \& Ribeiro (1996) em que, trabalhando com quatro subáreas e doze repetições de ensaio de infiltração em cada uma, o s(m) variou entre 14 e $35 \mathrm{~mm} \mathrm{~h}^{-1}$. Com relação ao IC, a MH contém $69,1 \%$ dos valores da VI (Tabela 3 ), sendo este o intervalo de confiança da $\mathrm{Md}$ os que apresentam maiores frequências.

Outras vantagens que reafirmam a VIP definida pela
MH em detrimento da Mo, são: valores menores do s, s 2 e CV. Essas medidas de dispersão apresentam restrições na escolha da VIP, pois sofrem forte influência dos valores extremos.

As velocidades de infiltração para projetos obtidos pelas técnicas das médias quadrática, cúbica e biquadrática, apresentaram-se inferiores às demais, na maioria dos atributos estudados; agora, a Média aritmética simples tornou-se a mais utilizada dentre as técnicas de definição; no entanto, se mostrou no presente trabalho, uma das mais deficientes, em virtude de se iniciar da distribuição de frequência, uma vez que a VIP está situada em uma classe que apresenta apenas $17,526 \%$ dos valores ( $T$ abela 2 ). Esta deficiência se explica pelo fato da VBI obedecer, nos ensaios realizados, a uma distribuição não normal; distribuições similares foram encontradas por Sales (1992), e Cichota et al. (2003), embora Vieira et al. (1981) e Araújo Filho \& Ribeiro (1996) tenham encontrado dados de distribuição normal; contudo, a VIP obtida pela MAS indicou algumas características que superaram as demais, como: s, s ${ }^{2}, \mathrm{~s}(\hat{\mathrm{m}})$ e CV (Tabela 1); os autores encontraram razão para essas superioridades em uma das propriedades fundamentais da MAS, ao afirmarem: “a soma dos quadrados dos desvios tomados em relação à média aritmética é a mínima possível"; com isto, se explica o fato, já que a base de cálculo do s é a soma dos quadrados dos desvios e a s ${ }^{2}, \mathrm{CV}$ e $\mathrm{s}(\hat{\mathrm{m}})$ são dependentes do $\mathrm{s}$.

Ficam validadas, então, as deficiências da VIP calculada pela MAS, na análise dos seguintes atributos: Dm e área de escoamento (Figura 2) - superior aos da Mo, MH, Md e MG; frequência de dados contido no IC da VIP - inferior à da Mo, MH, Md e MG (Tabela 3). Para Toledo \& Ovalle (1985), no caso de distribuição de frequências deformadas à direita (assimétrica) e se tratando de distribuição log normal, a média geométrica se revela mais apropriada que a MAS; a MG também é citada por Coelho et al. (1992) como uma das técnicas que mais se aproximam da velocidade real de infiltração do terreno.

Portanto, confirmando esses comentários e assumimos a MG como uma das mais apropriadas para a obtenção da VIP, pois ela apresentou Dm e áreas de escoamento (Figura 2) menores que as da MAS, MQ, Mc, Mb e frequência das VI no IC (Tabela 3) maior. Com relação à precisão da VIP obtida pela $\mathrm{MG}$, esta se apresentou superior à maioria das técnicas, com exceção da MAS. Os valores dos CV de todas as técnicas se apresentarem altos se comparados com os obtidos por Araújo Filho \& Ribeiro (1996) - 32\%; Silva (2003) - entre 11,97 e 37,80\% e Cichota et al. (2003) - 86,64\%; referidos valores podem ser explicados pela variabilidade natural do solo e pelas características da exploração agrícola da área: manejo intensivo do solo há vinte anos, compactação por transporte da safra e locais de realização dos ensaios - linha e entrelinha de cultivo.

Para Médici (1967) a utilização da Md é preferível à da MAS, quando os resultados extremos da série afetam sensivelmente o valor MAS; esta afirmação condiz com este estudo, já que os dados dos ensaios da VBI apresentaram valor mínimo e máximo de 3,33 e 282,02 $\mathrm{mm} \mathrm{h}^{-1}$, respectivamente, com uma At muito alta (T abela 1). 
O Dm ajuda não só a validar a conclusão de Médici, mas também, a preferência da Md sobre a MQ, Mc e Mb, pois o valor do desvio médio (média dos desvios da medida de tendência central em relação aos valores da série) da Md é muito semelhante ao da Mo, MH e MG e inferior ao das demais técnicas (Tabela 1).

Na comparação com a VIP da Mo e da MH, a da Md mostrou maior precisão, atribuída ao menor $\mathrm{s}(\mathrm{m})$, obtendose também, como bônus sobre todas as outras técnicas, exceto $\mathrm{MH}$, o IC contendo $69,1 \%$ da VI (Tabela 3). Verificou, na Figura 2, que a MG apresentou frequência superior na classe de $51,546 \%$ contra $17,526 \%$ com menor área de escoamento e maior frequência de valores contidos no IC.

\section{CONCLUSÕES}

1. A velocidade básica de infiltração indica distribuição $\log$ normal, tendo a distribuição de frequências assimetria à direita, deformação positiva.

2. A média aritmética simples nas condições do estudo não se caracteriza como aconselhável para tal, tornando necessários outros estudos com dados em distribuição normal.

3. A mediana e a média geométrica se mostram as mais recomendadas para a definição da velocidade de infiltração para projetos de irrigação por aspersão em áreas de Tabuleiros Costeiros, tendo a mediana apresentado o maior número de características desejáveis.

4. Na área estudada o valor da velocidade de infiltração para projetos de irrigação por aspersão se apresenta entre 37 e $44 \mathrm{~mm} \mathrm{~h}^{-1}$, que se configura na $\mathrm{Md} \pm \mathrm{s}^{2}(\hat{\mathrm{m}})$.

\section{LITERATURA CITADA}

Araújo Filho, J. C.; Ribeiro, M. R. Infiltração da água em cambissolos do baixio de Irecê - Bahia. Revista Brasileira de Ciência do Solo, v .20, p.363-370, 1996.

Bouwer, H. Intake rate: Cylinde infiltrometer . In: Klute, A. Methods of soil analysis. Part 1: Physical and mineralogical methods. Madson: American Society of Agronomy. Soil Science Society of American. 1986. 19p. Agronomy Series, 9

Brito, L. T. de L. Velocidades de infiltração determinadas por simulador de chuvas e por infiltrômetro de anel. Viçosa: UFV. 1994. 43p. Dissertação Mestrado

Calheiros, C. B. M.; Pereira, A. B.; Barbosa, S. C. S.; Bastos, A. L.; Silva, D. A. Velocidade básica de infiltração da água no solo (VBI Água) em solos das regiões Litoral Sul e Sul da Zona da Mata do Estado de Alagoas. Simpósio de Recursos Hídricos do Nordeste, 6, Maceió. Anais... Maceió: ANA/ UFAL, 2003. 3p.

Cichota, R.; Jong van Lier, Q.; Leguizamón Rojas, C. A. Variabilidade espacial da taxa de infiltração em Argissolo Vermelho. Revista Brasileira de Ciência do Solo, v .27, p.789-798, 2003.

Coelho, R. D.; Miranda, J. H. de; Duarte, S. N. Infiltração da água no solo: Parte I, Infiltrômetro de anéis versus infiltrômetro de aspersores. Revista Brasileira de Engenharia Agrícola e Ambiental, v.4, n.2, p.137-141. 2000a.
Coelho, R. D.; Miranda, J. H. de; Duarte, S. N. Infiltração da água no solo: Parte II, Acúmulo de água sobre a superfície do ter reno. Revista Brasileira de Engenharia Agrícola e Ambiental, v.4, n.2, p.142-145. 2000b.

Coelho, R. D.; Viccino, J. R.; Silva, V. M. Variabilidade espacial da infiltração de água no solo - infiltrômetro de anéis. In: Congresso Brasileiro de Engenharia Agrícola 1992, Campina Grande. Anais... Campina Grande: SBEA, 1992. 10p.

EMBRAPA - Empresa Brasileira de Pesquisa Agropecuária, Sistema brasileiro de classificação de solos. Rio de Janeiro: Embrapa/CNPS, 1999. 412p.

Kostiakov, A. N. "On the dynamics of the coef ficient of water percolation in soils and the necessity of studying it from dynamic point of view for purposes of amelioration." Trans. 6th Committee of International Society of Soil Science. Rússia. A15-21. 1932.

Leme, E. J. de A.; Uso da equação de Kostiakov para a seleção e adequação da intensidade de precipitação de aspersores. Brasília: Irrigação e Tecnologia Moderna (ITEM), n.31., p.37-40. 1987.

Lewis, M. R.; Power, W. L. A study of factors af fecting infiltration. Soil Science Sociedad American. v.3, p.334-339, 1938.

Lima, C. A. G.; Silans, A. P. de. Variabilidade espacial da infiltração de água no solo. Pesquisa Agropecuária Brasileira, v.34, n.12, p.2311-2320. 1999.

Médici, O. Curso de estatística geral. Bauru: Jalovi, 1967. 410p.

Parr, J. F.; Bertrand, A. R. Water infiltration into soil. Advances In Agronomy, v.12, p.311-342, 1960.

Pereira, W.; Tanaka, O. K. Elementos de estatística. São Paulo: McGraw-Hill do Brasil, 1984. 309p.

Sales, L. E. O. Variabilidade espacial da velocidade de infiltração básica associada a propriedades físicas das camadas superficial e subsuperficial de dois solos da região de Lavras (MG). Lavras: ESAL, 1992. 104p. Dissertação Mestrado

Santos, C. G. dos. Variabilidade espacial dos atributos químicos e físicos de solo cultivado com cana-de-açúcar em Coruripe, AL. Areia: UFPB, 2004. 64p. Dissertação Mestrado.

Shapiro, S. S.; W ilk, M. B. An analysis of variance test for normality (complete samples). Biometrika, v .52, p.591-611. 1965.

Silva, J. C. A. Infiltração da água no solo como indicador das modificações edáficas pelo sistema de manejo. Areia: UFPB, 2003. 72p.

Tenório, F. J. C; Calheiros, C. B. M. Técnicas para definição da velocidade básica de infiltração de projeto (VBIP), aplicada a solos do Estado de Alagoas. Congresso Brasileiro de Engenharia Agrícola, 23, São Pedro. Anais... São Pedro: SBEA, 2004. 6p.

Tenório, F. J. C.; Calheiros, C. B. M.; Madalena, J. A. da S.; Cunha, J. L. X. L.; Silva, J. A. C. da. Economia de água em irrigação por aspersão baseado na definição da velocidade de infiltração. Revista Caatinga, v .21 n.5, p.185-188, 2008.

Toledo, G. L.; Ovalle, I. I. Estatística básica. 2.ed. São Paulo: Atlas, 1985. 459p.

Vieira, S. R.; Hatfield, J. L.; Nielsen, D. R.; Biggar , J. W. Geoestatistical theory and application to variability of some agronomical properties. Hilgardia: Board, 1981. 73p. 